\title{
Resolving Range and Velocity Ambiguity Effectively and Efficiently with GPU
}

This paper was downloaded from TechRxiv (https://www.techrxiv.org).

\section{LICENSE}

CC BY 4.0

SUBMISSION DATE / POSTED DATE

$13-02-2022$ / 22-02-2022

\section{CITATION}

WANG, Zhifei (2022): Resolving Range and Velocity Ambiguity Effectively and Efficiently with GPU. TechRxiv. Preprint. https://doi.org/10.36227/techrxiv.19165892.v1

$\mathrm{DOI}$

10.36227/techrxiv.19165892.v1 


\section{Resolving Range and Velocity Ambiguity Effectively and Efficiently with GPU}

\author{
$1^{\text {st }}$ Zhifei WANG ${ }^{1,2}$ \\ 1. Key Laboratory of IntelliSense Technology, CET \\ 2. Nanjing Research Institute of Electronics Technology \\ Nanjing, China \\ zwangbc@connect.ust.hk
}

$3^{\text {rd }}$ Yuhao YANG ${ }^{1,2}$

1. Key Laboratory of IntelliSense Technology, CET

2. Nanjing Research Institute of Electronics Technology

Nanjing, China

\author{
$2^{\text {nd }}$ Junpeng $\mathrm{YU}^{1,2}$ \\ 1. Key Laboratory of IntelliSense Technology, CET \\ 2. Nanjing Research Institute of Electronics Technology \\ Nanjing, China
}

To gain better anti-error ability, clustering algorithm has

Abstract-Resolving the ambiguity in both range and velocity domain is one of the essential issues for the pulse Doppler (PD) radars using medium pulse repetition frequency (PRF) waveforms. The existing solutions are mainly based on the Chinese Remainder Theorem (CRT) and its variations and extensions, but these algorithms typically have an unsatisfactory accuracy and are highly complex in computation, especially for the case of a large false-alarm rate. In this work, we proposed a new approach to tackle with ambiguity based on the partial distance matrices (PDM) and implemented it on the NVIDIA graphics processing unit (GPU) platform. The evaluations based on both Monte-Carlo simulations and real measured data present the advantages of the proposed approach in accuracy and efficiency. The results also show the proposed approach can be utilized as a real-time algorithm even for a low-threshold detection scheme.

Index Terms - radar detection, ambiguity resolution, range and velocity ambiguity, medium PRF, GPU

\section{INTRODUCTION}

Medium pulse repetition frequency (PRF) waveforms are typically selected to balance the maximum unambiguous velocity and maximum unambiguous range of pulse Doppler (PD) radars. However, both range and velocity ambiguity will exist in this case. In order to resolve the ambiguity, multiple PRFs are employed to produce different ambiguous target range and velocity measurements.

Currently, the prevailing algorithm are mostly based on the Chinese Remainder Theorem (CRT) [1]-[3]. It is an analytic procedure for calculating the unambiguous range from the measured range on different PRFs. A graphical implementation of the CRT called coincidence algorithm and another variation of CRT referred to as residual look-up table (RLUT) have also been proposed in [4]-[7]. In these algorithms, the error in measurements is likely to degrade resolution results. In addition, a high computational complexity and a lot of memory space are required especially for the case where the interested range and velocity scopes are considerably large.

This work was supported by the National Key R\&D Program of China under Grant 2018AAA0102801. been proposed in [8], [9]. It rearranges all the possible ambiguous range corresponding to different PRFs in ascending order and calculate the cluster variation in order. The best cluster occurs at the value when the variation is a minimum. Although the clustering algorithm outperforms CRT, but it requires substantial computation resources, preventing it being applied in the practical real-time engineering.

Therefore, this work proposes a new ambiguity resolution approach based on partial distance matrices (PDM). We also implement the proposed algorithm on the NVIDIA graphics processing unit (GPU) platform with compute unified device architecture (CUDA). The evaluations show that the ambiguity in both range and velocity can be resolved effectively and efficiently. To our best knowledge, this is also the first time that an ambiguity resolution algorithm has been implemented on GPU and has exhibited the significant potential of GPU for the real-time practical engineering applications.

This paper is organized as follows. Section II states the ambiguity resolution problem, followed by the elaborations of the proposed algorithm with its implementation on GPU in Section III. IV evaluates and analyzes the proposed method with Monte-Carlo simulations and real measurements. Finally, $\mathrm{V}$ concludes the work.

\section{Problem Statement}

Assume a target is located at range $R$ and moves with radial velocity $v$. For PRF $i$, the measured range gate number $g_{i}$ by radar can be formulated as

$$
R=m_{i} R_{0, i}+\left(g_{f, i}+g_{i}\right) \Delta R+\delta R_{i},
$$

where $g_{0, i}$ and $R_{f, i}, \delta R_{i}$ are the maximum unambiguous range, the front gate number and range error for PRF $i$, respectively, $\Delta R$ is the range gate width, and $m_{i}$ is a nonnegative integer. The measured doppler gate $d_{i}$ satisfies

$$
v= \begin{cases}n_{i} v_{0, i}+d_{i} \Delta v+\delta v_{i}, & d_{i}=1,2, \ldots, \frac{N}{2} \\ n_{i} v_{0, i}+\left(d_{i}-N\right) \Delta v+\delta v_{i}, & d_{i}=\frac{N}{2}+1, \ldots, N\end{cases}
$$


where $v_{0, i}=\lambda f_{i} / 2$ is the maximum unambiguous velocity for PRF $i, \Delta v=\lambda f_{i} /(2 N)$ is the doppler gate width, $f_{i}$ is the value of the PRF, $\delta v_{i}$ is the velocity error, $N$ is the total doppler gate number of pulse processing, and $n_{i}$ is a positive integer.

Typically, the maximum unambiguous range of PRF $i$ is designed as certain multiple of range gate width, i.e. $R_{0, i}=$ $m_{0, i} \Delta R$, where $n_{0, i}$ is a positive integer. Consider a simple case where only one target is presented and no false-alarm exists, three PRFs are utilized to resolve the range ambiguity, and the velocity is unambiguous. Also assume there is no measurement error, i.e. $\delta R_{i}=0$ and $\delta v_{i}=0$. Then Eq. (1) can be reduced to

$$
R=\left(m_{i} m_{0, i}+\left(g_{f, i}+g_{i}\right)\right) \Delta R, \quad i=1,2,3 .
$$

The traditional CRT method gives the resolved range $R$ as

$$
\begin{aligned}
R= & \bmod \left(C_{1}\left(g_{f, 1}+g_{1}\right)+C_{2}\left(g_{f, 2}+g_{2}\right)\right. \\
& +C_{3}\left(g_{f, 3}+g_{3}\right), m_{0,1} m_{0,2} m_{0,3} \Delta R,
\end{aligned}
$$

where the variables $C_{i}$ are constants related to $m_{0, i}$, and the details can be found in [1].

It should be noted that the calculation in CRT based on discrete range gates, causing possible errors if measurement errors exist or the target is located at the margin of a range gate. Other errors in the system, such as the errors in PRFs, front range gate number etc., will lead to performance degradation of CRT. Furthermore, if there exist more than one target and some false alarms, the computational complexity grows explosively.

The graphic version of CRT can mitigate the complexity to some extent, but a large memory space to store the rangevelocity information of all target candidates will be searched frequently, slowing down the processing speed. The computational complexity reaches $O\left(N_{r} N_{v}\right)$, where $N_{r}$ and $N_{v}$ are the number of total range gates and doppler gates of the maximum range and velocity of interest, respectively. For example, if maximum range of interest is $200 \mathrm{~km}$ and velocity of interest ranges from $-300 \mathrm{~m} / \mathrm{s}$ to $300 \mathrm{~m} / \mathrm{s}$, and the range resolution is $30 \mathrm{~m}$ and the velocity resolution is $1 \mathrm{~m} / \mathrm{s}$, then it requires at least $2 \times 10^{5} / 30 \cdot 600 \approx 3.8 \mathrm{MB}$ memory space for one PRF. If there are 20 candidates in the unambiguous range, and the maximum range of interest is 30 times of the unambiguous range, then at least 1200 indexing and assignment followed by $4 \times 10^{6}$ addition operations are required for the ambiguity resolution with two PRFs and at least $4 \times 10^{6}$ sequential search operations are demanded. This prevents an efficient ambiguity resolution implementation.

\section{Solving Ambiguity based on PARTIAL Distance MATRICES}

The problem of resolving ambiguity can be treated as the problem of matching. The ambiguous measurements in range and velocity firstly are extended to different ambiguous regions according to Eq. (1) and (2), respectively. The generated possible targets with the extended range and velocity together with the original ambiguous measurements of targets are

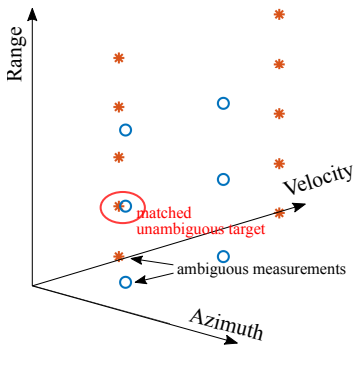

(a)

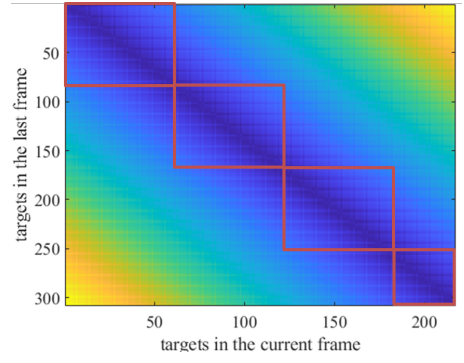

(b)
Fig. 1: (a) is the concept of resolveing range and velocity ambiguity with the distance matrix, and (b) example of a distance matrix and partial distance matrices.

called target candidates. Then the similarity of the target candidates of each PRF can be calculated. If any similarity is over a threshold, the corresponding candidates are from the measurements of one target, and the range and velocity of the real target can be determined.

In this work, we utilize the distance of target candidates of different PRFs to evaluate their similarity. The difference in range and velocity are utilized to evaluate the distance. Information in other dimensions can also be leveraged to calculate distance and evaluate similarity. In order to use multiple PRFs to resolve ambiguity, the beamwidth should be sufficient large to cover the space in the sequential several frames. Therefore, we also use the measured angle to differentiate the real target from false alarms. Mathematically, the test of a target can be formulated as

$$
D\left(R_{i}, v_{i}, \theta_{i} ; R_{j}, v_{j}, \theta_{j}\right) \stackrel{\stackrel{H_{0}}{>}}{\underset{H_{1}}{<}} T h,
$$

where $D(\cdot)$ is the distance function of two candidates, and any target candidate $p_{i}$ is described by its range, velocity and azimuth, i.e. $R_{i}, v_{i}, \theta_{i}, T h$ is the threshold, and $H_{1}$ and $H_{0}$ represents the hypotheses that these two candidates are matched and mismatched, respectively.

To reduce the computational complexity, we use the distance function as below.

$$
\begin{aligned}
& D\left(R_{i}, v_{i}, \theta_{i} ; R_{j}, v_{j}, \theta_{j}\right)= \\
& \quad \begin{cases}\infty, & \left|v_{i}-v_{j}\right| \geq v_{t h} \text { or }\left|\theta_{i}-\theta_{j}\right| \geq \theta_{t h}, \\
\left|R_{i}-R_{j}\right|, & \left|v_{i}-v_{j}\right|<v_{t h} \text { and }\left|\theta_{i}-\theta_{j}\right|<\theta_{t h},\end{cases}
\end{aligned}
$$

where $v_{t h}$ and $\theta_{t h}$ are the threshold of velocity and azimuth, respectively.

Fig. 1(a) shows a simple example. For simplicity, ambiguous measurements of two different PRFs are considered, and only one target is detected for each PRF. In the figure, blue circles and red stars represent the target candidates extended from the ambiguous measurements of two targets of two different PRFs, respectively. The extension is conducted in both range and velocity domains. The distance of two target candidates marked inside the red circles is smaller than the distance 

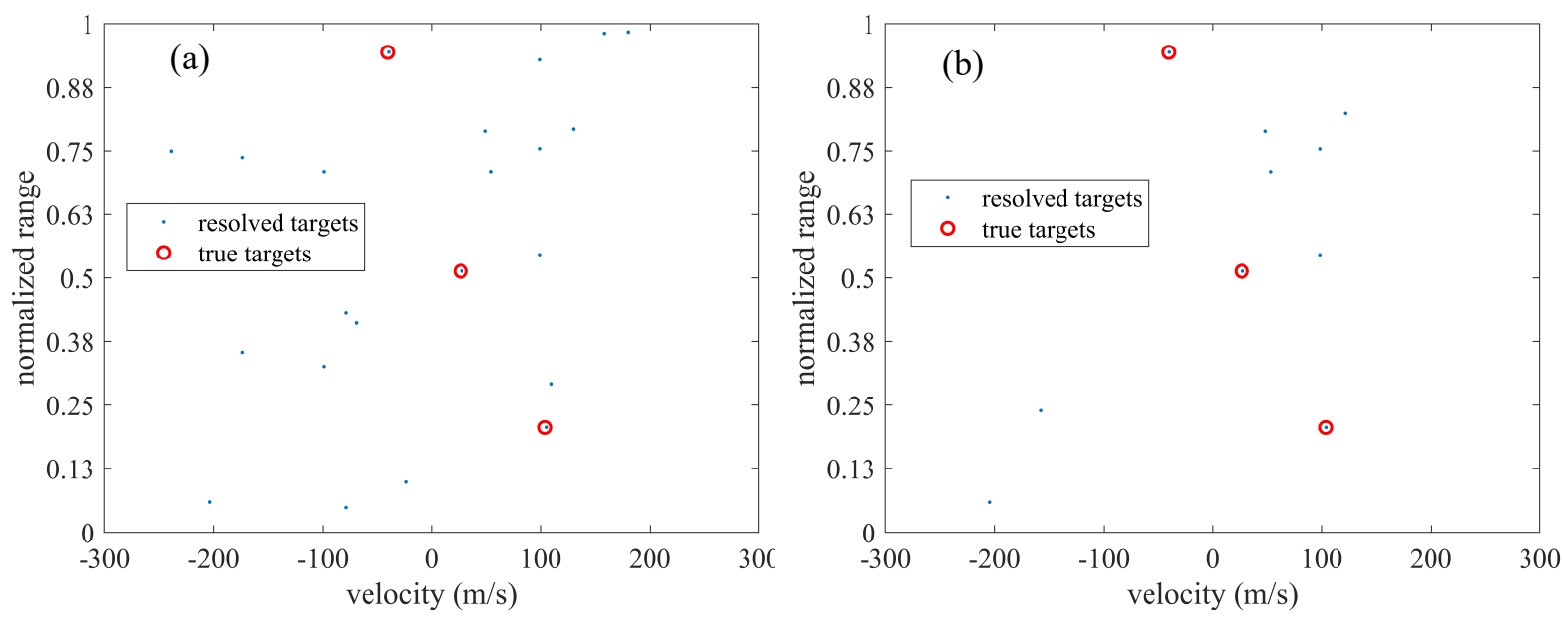

Fig. 2: Ambiguity resolution results of (a) the conventional CRT and (b) the proposed PDM based on Monte-Carlo simulations.

threshold. Thus, they are determined to be matched, i.e. the unambiguous measurements of one target under two PRFs. The range and velocity of the target are the weighted average of the range and velocity of these two candidates.

Directly using distance matrix to resolve ambiguity also demands for considerable resources. The space and computational complexity both are as large as $O\left(N^{2}\right)$, where $N$ is the average number of ambiguous measurements per PRF. Consider the aforementioned example in Section II. Resolving ambiguity requires $4.32 \mathrm{MB}$ memory space for single-float precision, and at least $1.08 \times 10^{6}$ subtraction and $3 \times 10^{5}$ search operations are demanded.

To further reduce the computation and memory complexity, we divided all the target candidates into several blocks according to the range of the target candidates. Instead of calculating the distance among all the candidates, calculating the distance between any two candidates in the same block is sufficient. In other words, the calculation of the whole distance matrix will be reduced to the calculation of parts of the original distance matrix, referred as the partial distance matrices in this work. Fig. 1(b) illustrates an example of the value of a distance matrix, and the regions with red box are partial distance matrices. For simplicity, the velocity and azimuth of all the candidates are assumed the same.

Assume the target candidates are located uniformly in the whole range scope in the aforementioned example, and the range scope of a partial region is $10 \mathrm{~km}$. Then, there will be 20 blocks, and only 30 candidates exist in each block. In consequence, the required memory space will be reduced to $216 \mathrm{~KB}$, the subtraction operations will be reduced to $5.4 \times$ $10^{4}$, and the search operations will be reduced to $1.8 \times 10^{4}$. In general, both of the space and computational complexity are reduced to $O\left(N^{2} / M\right)$, where $M$ is the number of partial distance matrices.

It is worth noting that generating partial distance matrices can be fully parallelized, and extending ambiguous targets to all possible range and velocity according to Eq. 1 and 2 and extracting matched targets can also be partially parallelized. This makes GPU extremely suitable for ambiguity resolution with PDM. GPUs excel processing of a large amount of data by using the same set of instructions, referred to as single instruction multiple data (SIMD) technology. For example, the $5.4 \times 10^{4}$ subtraction can be performed simultaneously, thus greatly speeding up calculation.

Due to page limit, we briefly introduce the implementation of our proposed algorithm on GPU platform with CUDA as follows. When extending ambiguous targets, the thread dimension is set to the number of measured targets, and the grained dimension is set to the number of extending times. Meanwhile, all the target candidates are divided into different blocks according to their range, preparing for the calculation of partial distance matrices. Here, a vital technology called warp aggregated atomics using cooperative groups (CG) will be utilized to realize a lock when arrange all candidates in parallel. Then, another kernel generates partial distance matrices between two different PRFs with the grained dimension of the number of the target candidates of the current frame and the block dimension of the number of the candidates of the last frame. Next, the minimum of each column is selected in parallel. Two candidates are determined to be matched if the minimum is smaller than the threshold. Finally, the unambiguous target information will be extracted.

\section{EVALUATIONS AND ANALYSES}

We first evaluate the performance of the proposed PDM based on Monte-Carlo simulations. The comparison with a graphic version of CRT, the coincidence algorithm discussed in [10], is conducted. To overcome measurement errors, a tolerance of one range gate is established. This means that a target is declared if a detection occurs in all PRFs at some range gate $g \pm 1$. At each iteration of the simulations, the range and velocity of targets are generated in the first step. The number of targets is a Gaussian random variable ranging from 1 to 5 . Given the values of PRFs, range gate width and doppler 

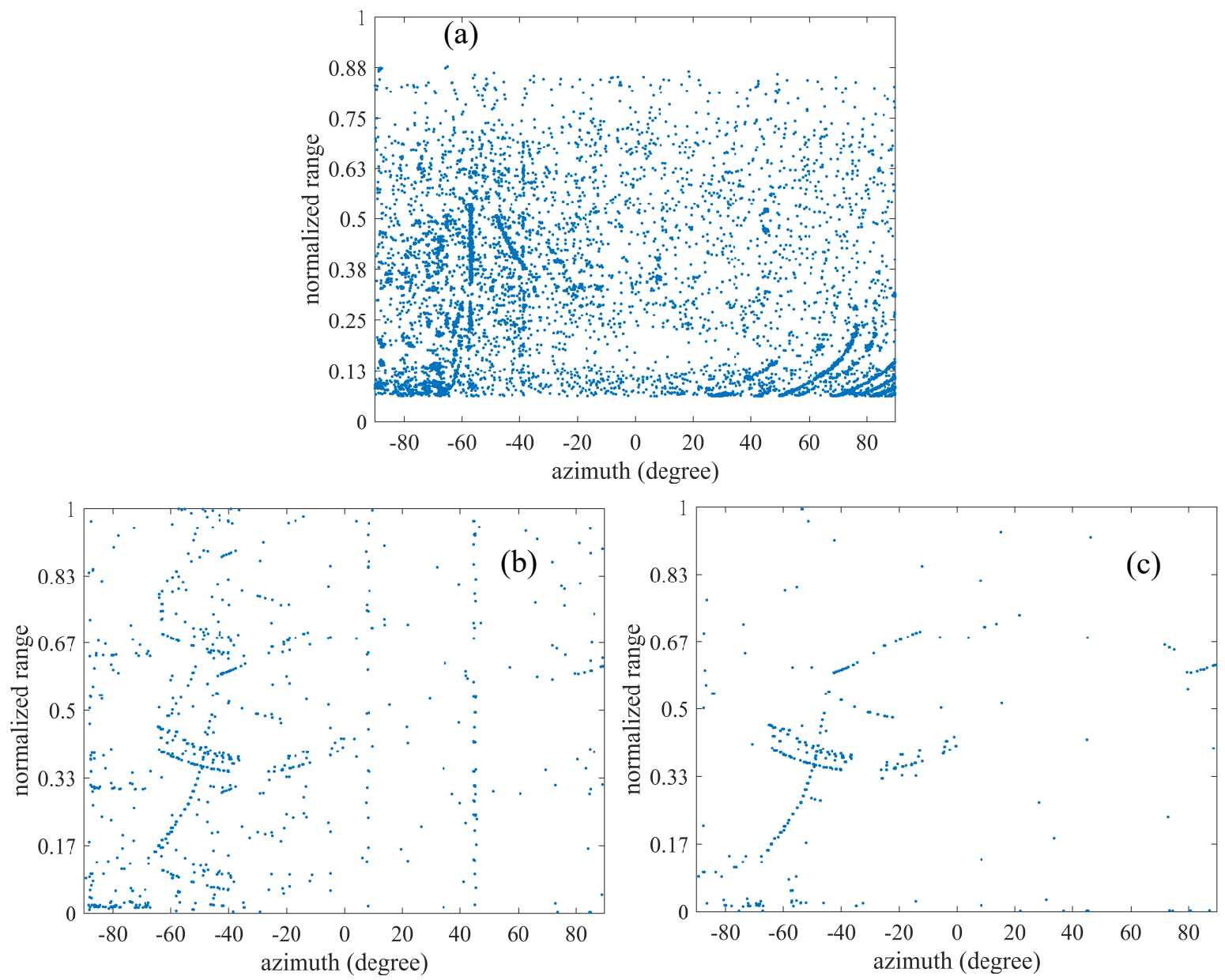

Fig. 3: Ambiguity resolution results of the conventional CRT and the proposed approach based on simulations.

TABLE I: Monte-Carlo simulation results of the conventional CRT and the proposed PDM.

\begin{tabular}{c|cc}
\hline Method & $\begin{array}{c}\text { Average } \\
\text { detection rate }\end{array}$ & $\begin{array}{c}\text { Average } \\
\text { false-alarm rate }\end{array}$ \\
\hline CRT & 0.9267 & $1.93 \times 10^{-6}$ \\
Proposed & 0.9642 & $6.92 \times 10^{-7}$ \\
\hline
\end{tabular}

gate width, the ambiguous range gates and doppler gates of the targets can be obtained. Here, random errors in range and velocity in Gaussian distribution will be added. Meanwhile, some false alarms will be generated randomly as well. Here, we use two different PRFs to resolve the range and velocity ambiguity. Fig. 2 shows the results of the two methods at one iteration in the Monte-Carlo simulations. Table I shows the detection rate and false alarm rate after ambiguity resolution of 5000-iteration Monte-Carlo simulations.

As shown in Fig. 2, both PDM and CRT can resolve the range and velocity ambiguity correctly in this case. However, the false alarms of PDM are noticeably fewer than those of CRT, which is supported by the statistics of the MonteCarlo simulations as shown in Table I. The false alarm rate of ambiguous measurements is around $5.38 \times 10^{-4}$ before resolution. Both PDM and CRT can reduce the false alarm rate after ambiguity resolution, since the resolution process can be treated as binary integration. However, in addition to the true target, a series of false targets also appear periodically at the same velocity of the true target in the results of CRT, while no such phenomenon exists in the results of PDM. This results from a looser matching condition in CRT due to the existence of the one tolerance gate. As shown in Table I, compared to CRT, PDM achieves over $4 \%$ improvement in detection rate and over $64 \%$ reduction of false alarm rate.

Apart from Monte-Carlo simulations, we also evaluate the proposed PDM and compared it with CRT in real applications. Fig. 3(a) shows the ambiguous measurements of a groundbased radar. Four sequential frames with different PRFs are utilized to resolve the range and velocity ambiguity of an area. The range is normalized to the maximum unambiguous range. As we can note, identifying targets is almost impossible in this case, due to the small maximum unambiguous range and high speed of the targets. 


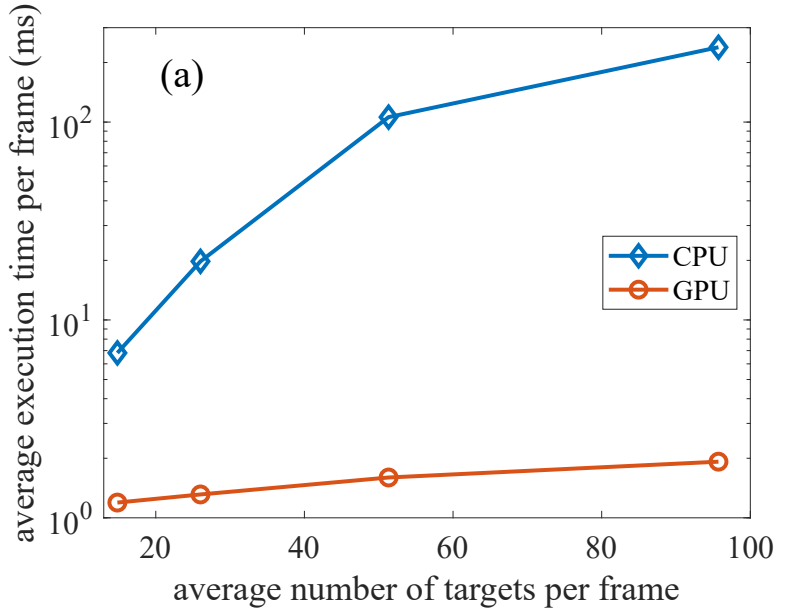

Fig. 4: Processing time of PDM algorithm on CPU and GPU platform. (a) is the average processing time per frame, and (b) is the average processing time per detected target.

Fig. 3(b) and 3(c) show the ambiguity resolution results of CRT and our proposed PDM algorithm, respectively. Both algorithms can accomplish to obtain unambiguous targets. However, more false alarms appear when using CRT. PDM algorithm is capable of resolving ambiguity remarkably better than CRT. This agrees well to the results of Monte-Carlo simulations. The improvement stems from using range and velocity information in the continuous spaces in PDM rather than the discrete ones in CRT. In other words, a finer-grained information is exploited in PDM. Another important reason is the utilization of additional information in azimuth domain.

We also assess the processing time of PDM algorithm on CPU platform and GPU platform. The results are shown in Fig. 4. The number of detected ambiguous targets per frame is changed by adjusting detection threshold. It should be noted that the processing time of PDM on CPU platform grows rapidly with the average number of ambiguous targets per frame, while that of PDM on GPU platform only slightly increases. Moreover, the average processing time per detected target even decreases with the number of targets per frame, mainly because the overhead of launching CUDA kernels and sequential operations are amortized. This also proves that PDM has been effectively accelerated by the parallelism of GPU platform.

The average frame time is in the order of 10s microseconds. This indicates that CPU-based PDM can be used as a realtime ambiguity resolution algorithm if the detection threshold is relatively high. Even if low-threshold detection is adopted, the average processing time of GPU-based PDM can be well limited to within $2 \mathrm{~ms}$, making it highly efficient in real applications.

\section{CONCLUSION}

This work has proposed a new ambiguity resolution algorithm based on partial distance matrices (PDM). The proposed algorithm exploits fine-grained range and velocity information

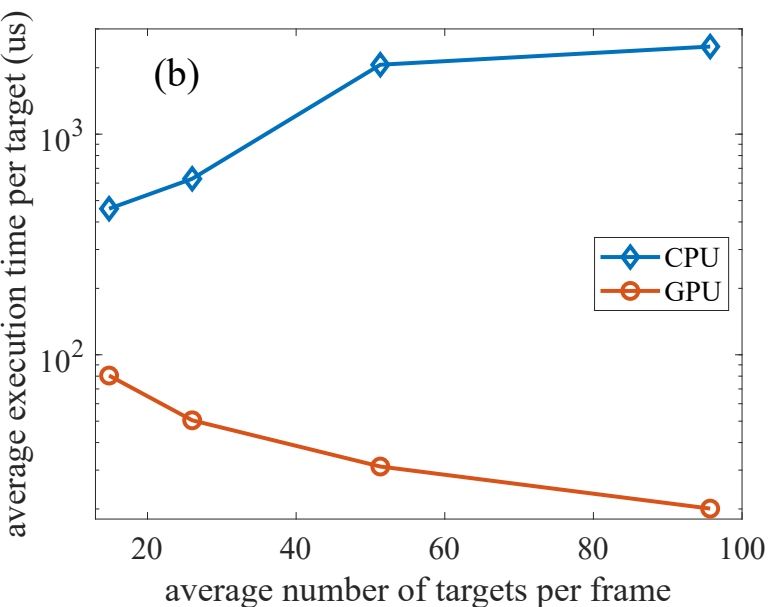

and additional information in azimuth domain, facilitating its capability of high ambiguity resolution accuracy, strict false alarm suppression and insensitive to measurement errors. Extensive analyses present the small complexity of PDM. The parallel implementation of PDM on GPU platform accelerates the ambiguity resolution process, ensuring the average execution time per frame below 2 ms even for a low-threshold detection and paving a way for the practical real-time engineering applications.

\section{REFERENCES}

[1] Z. Huang and Z. Wan, "Range ambiguity resolution in multiple prf pulse doppler radars," in ICASSP'87. IEEE International Conference on Acoustics, Speech, and Signal Processing, vol. 12, pp. 1786-1789, IEEE, 1987.

[2] W. Dai, J. Wang, and J. Zeng, "The error detecting and error-correcting conditions of the range ambiguties solved by chinese remainder theorem," Acta Electronica Sinica, p. 06, 1988.

[3] D. Nagel and H. Hommel, "A new HPRF mode with highly accurate ranging capability for future airborne radars," in 2001 CIE International Conference on Radar Proceedings (Cat No. 01TH8559), pp. 275-280, IEEE, 2001.

[4] S. Hovanessian, "An algorithm for calculation of range in a multiple PRF radar," IEEE Transactions on Aerospace and Electronic Systems, no. 2, pp. 287-290, 1976.

[5] G. Trunk and S. Brockett, "Range and velocity ambiguity resolution," in The Record of the 1993 IEEE National Radar Conference, pp. 146-149, IEEE, 1993.

[6] W. Lei et al., "The resolution of range ambiguity in a medium pulse Doppler radar," Journal of Beijing Institute of Technology, vol. 19, no. 3, pp. 357-360, 1999.

[7] W. Qiu et al., "Improvements for range and speed ambiguity resolving 2007.

[8] W. Lei, T. Long, and Y. Han, "Resolution of range and velocity ambiguity for a medium pulse Doppler radar," in Record of the IEEE 2000 International Radar Conference [Cat. No. O0CH37037], pp. 560564, IEEE, 2000.

[9] M. Li and M. Li, "High efficiency algorithm of PD radar for range ambiguity resolution based on the one-dimension method," Electronic Information Warfare Technology, vol. 5, p. 004, 2010.

[10] M. A. Richards et al., Principles of modern radar volume I - basic principles. Citeseer, 2010. in PD mode," Ship Electronic Engineering, vol. 27, no. 4, pp. 100-102, 